Document downloaded from:

http://hdl.handle.net/10251/111608

This paper must be cited as:

Barjau, C.; Fuentes, M.; Shitomi, T.; Gomez-Barquero, D. (2017). MIMO Sphere Decoding With Successive Interference Cancellation for Two-Dimensional Non-Uniform Constellations. IEEE Communications Letters. 21(5):1015-1018. doi:10.1109/LCOMM.2017.2653775

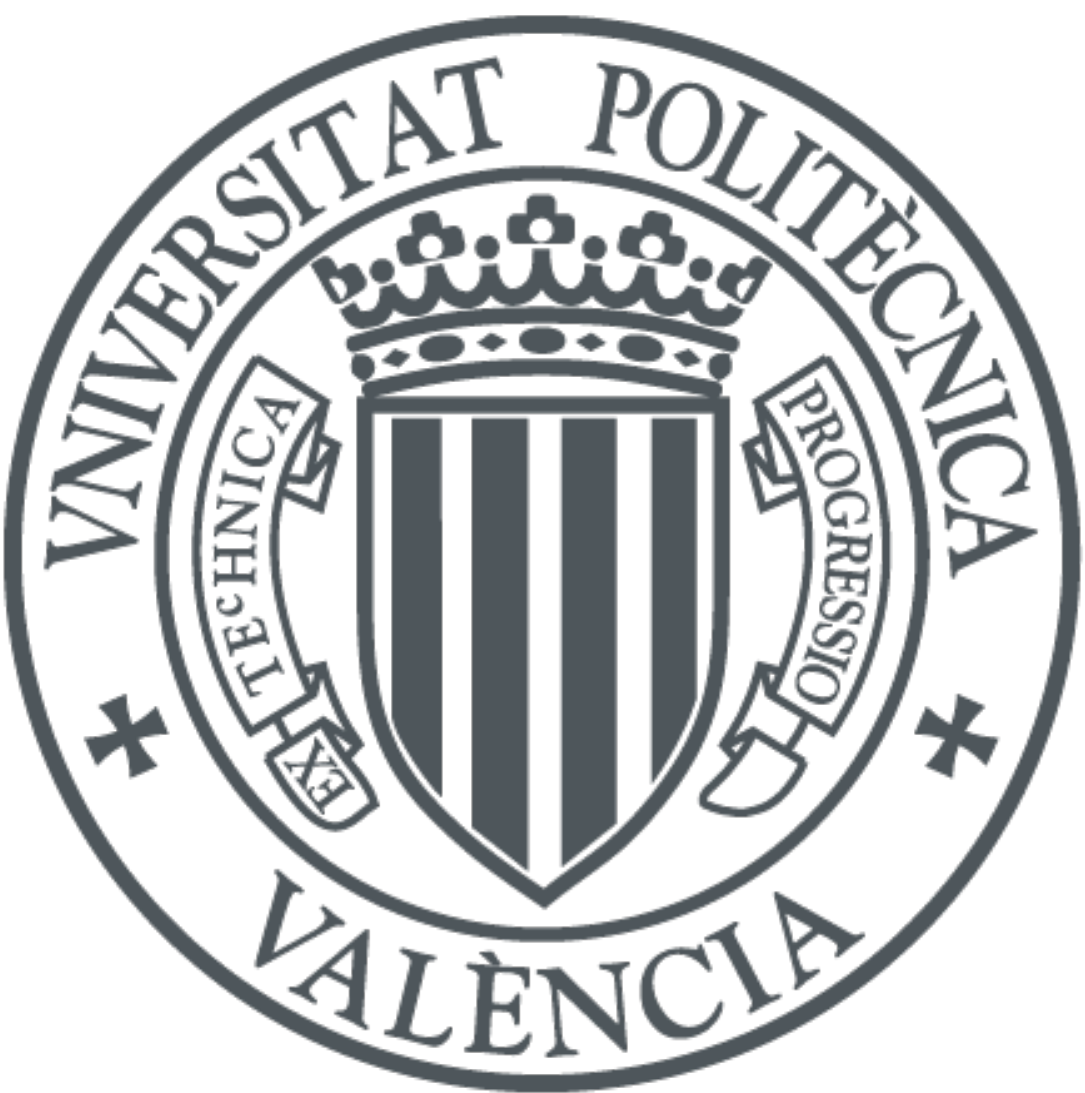

The final publication is available at

https://doi.org/10.1109/LCOMM.2017.2653775

Copyright Institute of Electrical and Electronics Engineers

Additional Information 


\title{
MIMO Sphere Decoding with Successive Interference Cancellation for Two-Dimensional Non-Uniform Constellations
}

\author{
Carlos Barjau, Manuel Fuentes, Takuya Shitomi and David Gómez-Barquero
}

\begin{abstract}
Non-Uniform Constellations (NUCs) have been introduced to improve the performance of QAM constellations. 1D-NUCs keep the squared shape, while 2D-NUCs break that constraint to provide robustness. An impending problem with Multiple-Input Multiple-Output (MIMO) is the optimum demapping complexity, which grows exponentially with the number of antennas and the constellation order. Some well-known suboptimum MIMO demappers such as Soft Fixed-Complexity Sphere Decoders (SFSD) can reduce that complexity. However, SFSD demappers do not work with 2D-NUCs, since they perform a quantization step in separated $I / Q$ components. In this letter, we provide an efficient solution for $2 \mathrm{D}$-NUCs based on Voronoi regions. Both complexity implications and SNR performance are also analyzed.
\end{abstract}

Index Terms-ATSC 3.0, Non-Uniform Constellations, MIMO, Sphere Decoder, Successive Interference Cancellation.

\section{INTRODUCTION}

$\mathbf{N}$ ON-UNIFORM Constellations (NUCs) have been recently introduced in new-generation Digital Terrestrial Television (DTT) systems to reduce the gap between the BICM (Bit-Interleaved Coding and Modulation) capacity of uniform Gray-labelled constellations and the unconstrained Shannon limit [1]. Compared to uniform QAM (Quadrature Amplitude Modulation) constellations, NUCs improve the transmission robustness, reducing the Signal-to-Noise Ratio (SNR) necessary to guarantee a particular Bit Error Rate (BER). NUCs provide a coverage gain, although it can be translated into a capacity (spectral efficiency) gain.

NUC symbols are optimized by means of signal shaping techniques for a particular SNR and channel model [2], while keeping an average transmit power normalized to unity. NUCs are categorized into two different groups: one-dimensional and two-dimensional NUCs (1D-NUC and 2D-NUC, respectively). 1D-NUCs have a squared shape with non-uniform distance between constellation symbols. In this case, both components can be separated, since in-phase (I) and quadrature (Q) components use identical and independent PAM mappings. 2D-NUCs are designed by relaxing the square shape constraint, with a better SNR performance than 1D-NUCs but with a higher demapping complexity, since a 2D-demapper is required.

NUCs have been used in several broadcasting systems. DVB-NGH (Digital Video Broadcasting - Next Gen. Hand-

This work was partially supported by the Ministry of Economy and Competitiveness of Spain (TEC2014-56483-R), co-funded by the European FEDER fund.

C. Barjau, M. Fuentes and D. Gómez-Barquero are with the Institute of Telecommunications and Multimedia Applications (iTEAM) of the Universtat Politecnica de Valencia, Valencia, Spain (e-mail: \{carbare1, mafuemue, dagobar\}@iteam.upv.es).

T. Shitomi is with the Science and Technology Research Laboratories of Japan Broadcasting Corporation (NHK), Tokyo, Japan (e-mail: shitomi.tgy@nhk.or.jp).
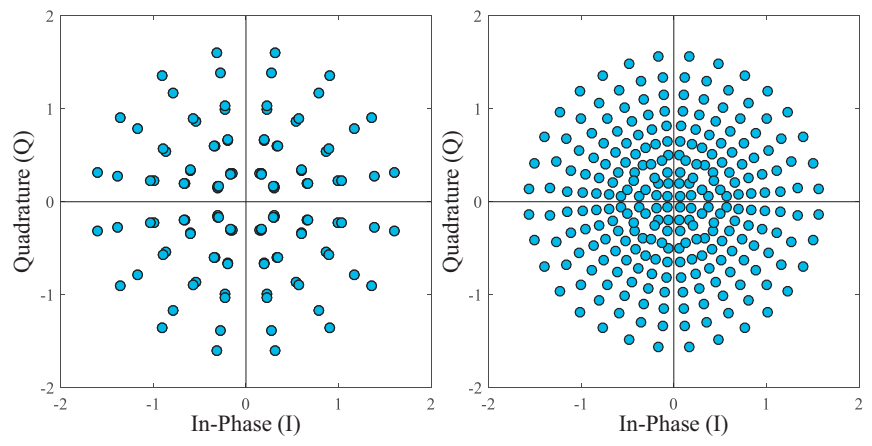

Fig. 1. Examples of 2D-256NUC constellations optimized for SNRs of 10 $\mathrm{dB}$ (left) and $20 \mathrm{~dB}$ (right), for i.i.d. Rayleigh channel.

held) was the first to include 1D-NUCs for 64- and 256point constellation orders [3]. The new U.S. terrestrial broadcasting standard ATSC 3.0 [4] (Advanced Television Systems Committee 3rd Generation) adopted 2D-NUCs from 16 to $256 \mathrm{NUC}$, and $1 \mathrm{D}-\mathrm{NUCs}$ for $1024 \mathrm{NUC}$ (or $1 \mathrm{kNUC}$ ) and 4096NUC (or 4kNUC) [5], for both SISO (Single-Input Single-Output) and MIMO (Multiple-Input Multiple-Output) antenna profiles [6].

Results in [5] show that the gain of 2D-NUCs compared to QAM increases with the order of constellation, being maximum for medium code rates (CR). For example, in ATSC 3.0 , a maximum gain of $1.3 \mathrm{~dB}$ is achieved for $256 \mathrm{NUC}$ and a CR 7/15, for AWGN channel. Fig. 1 depicts two 2D-NUCs optimized for SNRs of 10 and $20 \mathrm{~dB}$ in an i.i.d. (independent and identically distributed) Rayleigh channel. It can be noted that constellations retain up-down and left-right symmetry. When designing NUCs, the bits related to left-most labels offer more robust capacities. For that reason, at low SNRs (see the left side of Fig. 1), NUCs collapse into lower order constellations and several symbols are almost identical. Due to this condensation, these bits have a robustness similar to the equivalent lower order constellation, and the bits related to right-most labels cannot be resolved from the overlapping points, since they provide weak information [5].

The use of NUCs with MIMO is a relatively new research topic in the literature. In [7] and [8], it was shown that NUCs can be efficiently combined with multi-antenna MIMO systems. However, an impending problem is the extremely high demapping complexity when using optimum MaximumLikelihood (ML) or max-log demappers. In this case, the number of possible received symbols grows exponentially with the number of antennas and the constellation order. To reduce this complexity, Soft Fixed-Complexity Sphere Decoders (SFSD) can be used, which achieve a sub-optimum max-log performance [9]. With SFSD, it is necessary to per- 
form a quantization step called Zero Forcing with Successive Interference Cancellation (ZF-SIC) that needs to be performed in separated in-phase (I) and quadrature (Q) components [10]. With 1D-NUCs, thanks to the squared shape, both components can be separated. However, this is not possible with 2D-NUCs.

In this paper, we propose an efficient pre-processing approach for 2D-NUCs based on the Voronoi regions for the ZF-SIC quantization step used in SFSD, which has never been done in the current literature, according to the best of authors' knowledge. Throughout the paper, the term ZF-SIC is used to refer to the quantization step in the SFSD demapper and not the equalization process. The proposed technique is compared with the optimum ML and max-log demappers in terms of visited nodes and system performance. The proposed method obtains up to $97.5 \%$ of complexity reduction using 64NUCs, with a degradation lower than $0.1 \mathrm{~dB}$ and $0.7 \mathrm{~dB}$ compared to max-log and ML respectively. While results focus on the new ATSC 3.0 standard, the conclusions reached in this article for 2D-NUCs and MIMO apply to all mobile communication systems, including unicast point-to-point transmissions.

The paper is structured as follows. Section II describes the background of ZF-SIC techniques applied to MIMO, and the quantization problem when using 2D-NUCs. In Section III, we propose and explain a method for the quantization step. Section IV compares the proposed method with ML and max-log demappers, in terms of complexity reduction and SNR performance. Finally, the main findings of the work are summarized in Section V.

\section{BACKGROUND}

In MIMO systems, ZF-SIC is a particular subset of linear detectors [10]. The MIMO channel model employed in this paper is shown in Eq. 1.

$$
\mathbf{y}=\mathbf{H s}+\mathbf{n}
$$

where $\mathbf{y}$ is the received vector, formed by $\left(y_{1}, \ldots, y_{N_{R}}\right)$ symbols, $\mathbf{H}$ is the channel realization matrix, with $N_{R}$ rows and $N_{T}$ columns, $N_{R}$ and $N_{T}$ are the number of receiving and transmitting antennas, $\mathbf{s}$ is a vector $\left(s_{1}, \ldots, s_{N_{T}}\right)$ with possible transmitted constellation symbols, and $\mathbf{n}$ is the noise vector $\left(n_{1}, \ldots, n_{N_{R}}\right)$. In this paper, $N_{R}$ is assumed equal to $N_{T}$.

ZF-SIC calculates the QR decomposition of the channel realization matrix $\mathbf{H}$ [11], where $\mathbf{Q}$ and $\mathbf{R}$ are an orthogonal and triangular matrix, respectively. It iteratively obtains an estimate of the received component from a single antenna. The estimated symbol will be used to obtain the components of the remaining antennas, as shown in Eq. 2.

$$
\hat{s}_{m}=\Gamma\left\{\frac{z_{m}-\sum_{i=m+1}^{N_{R}} R_{m, i} \hat{s}_{i}}{R_{m, m}}\right\}, m=N_{R}, \ldots, 1
$$

where $\hat{s}_{m}$ is the quantified estimated symbol from the $m^{\text {th }}$ antenna, $z_{m}$ is the received symbol multiplied by $\mathbf{Q}^{H}$, and $(\cdot)^{H}$ refers to the Hermitian operation. $\Gamma$ refers to the function that gives the quantized value from a continuous input. With QAM and 1D-NUCs, estimated complex symbols are separated into real and imaginary parts, and associated to the nearest value

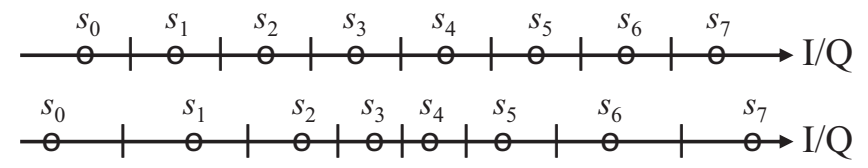

Fig. 2. Example of quantization for I/Q components of 64QAM (top) and 1D-64NUC (bottom)

in each dimension, as shown in Fig. 2. The only difference using QAM or 1D-NUCs is the use of a uniform or a nonuniform quantizer. However, using 2D-NUCs, the quantization is no longer direct, since constellations do not have a squared shape and I/Q components cannot be separated. A new method is required, in order to allow ZF-SIC to work with these new constellations, and is presented in the following section.

\section{VORONOI REgIONS SELECTION FOR AN EFFICIENT FIXED SPHERE DECODING WITH 2D-NUC}

In mathematics, a Voronoi diagram is a partitioning of a plane into regions based on distances to the points in a specific subset of the plane [12]. For each constellation symbol, there is a corresponding Voronoi region in the constellation diagram consisting of the closest points to that symbol. Let $X$ be a metric space with distance function $d$. Let $I$ be a set of indexes and let $\left(P_{i}\right)_{i \in I}$ be an ordered collection of symbols in the space $X$. The Voronoi region $R_{i}$ associated to the symbol $P_{i}$ is the set of all points in $X$ whose distance to $P_{i}$ is lower than their distance to the other symbols $P_{j}$, where $j$ is any index different from $i$. This can be seen in Eq. 3. The Voronoi diagram is simply the tuple of cells $\left(R_{i}\right)_{i \in I}$.

$$
R_{i}=\left\{x \in X \mid d\left(x, P_{i}\right) \leq d\left(x, P_{j}\right), j \neq i\right\}
$$

The proposed method is based on this mathematical approach, and is called Voronoi Regions Selection (VRS). The algorithm consists of two different phases: the first phase calculates a Look-Up Table (LUT), from the Voronoi regions associated to a given NUC. This phase is only done once, before the communication takes place, and the LUT gets stored at the receiver. In the second phase, the receiver, which is already running, loads the LUT in order to receive the signal with VRS and SFSD demapping. In practice, the LUT is equivalent to an image, where each pixel has an integer value associated. The received symbol is assigned to a particular pixel, and the corresponding value is related to a constellation symbol, as shown in Fig. 3. Since constellations retain updown and left-right symmetry, only the first quadrant needs to be stored. The other three quadrants can be derived by just changing the sign of real and imaginary parts of the received symbol. A LUT with squared shape has been selected as a compromise to accommodate different distributions of NUC constellation symbols, for practical implementations in real receivers. The LUT corresponding to every possible 2DNUC used in a certain specification, e.g. ATSC 3.0, could be calculated beforehand and uploaded to the receiver memory.

The memory usage for a LUT depends on two different variables: the number of cells or pixels in one of the two $\mathrm{I} / \mathrm{Q}$ axis considered $(k)$, which determines the total LUT 


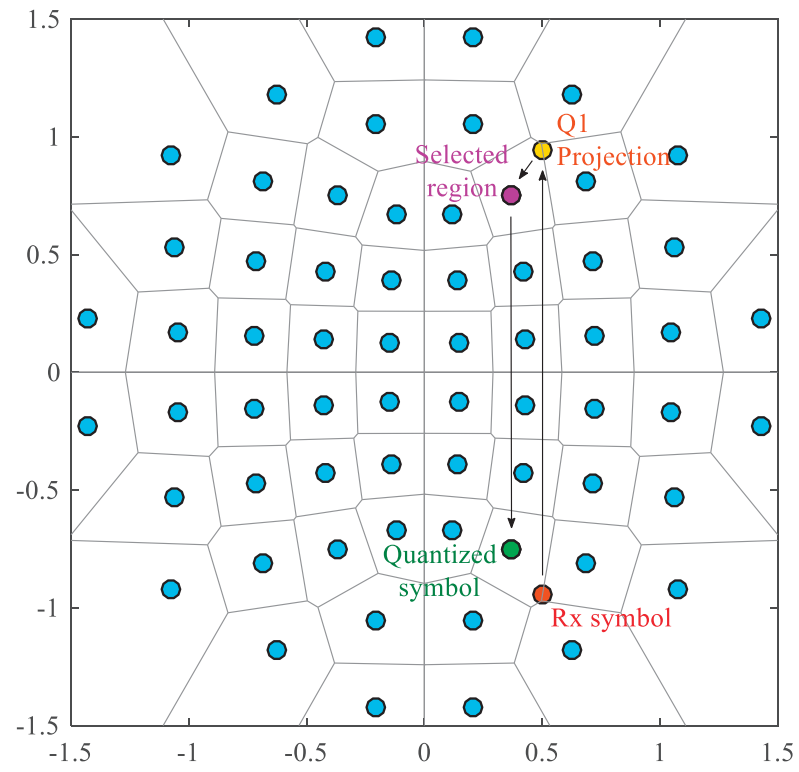

Fig. 3. Example of Voronoi regions calculated for a 2D-64NUC, optimized for a SNR of $20 \mathrm{~dB}$.

size $(k \times k)$, and the number of bits $(b)$ assigned to each value/pixel of the LUT stored. The parameter $b$ is directly associated to the constellation size and only has memory implications. The maximum number of regions to store is a quadrant of the constellation, i.e. $M / 4$, being $M$ the number of symbols. Hence, the amount of bits can be calculated as $\left\lceil b=\log _{2}(M / 4)\right\rceil$. For instance, using a non-condensed 64NUC with $b=4$ and $k=40$, the stored LUT requires 6.40 Kbits. In case of condensation, the number of regions can be further reduced, as proposed in [13]. For example, using a 64NUC optimized for a CR 2/15, the number of regions is reduced to $M=4$, so the number of bits required is only $b=2$.

To calculate the LUT, three steps are required. In the first step, the constellation symbols are grouped in clusters and removed, since they hinder the calculation of the Voronoi Regions. This step introduces an insignificant loss in performance, since lineal Euclidean distances among condensed symbols in normalized NUCs are almost negligible, lower than $10^{-2}$ [13]. In order to detect clusters, an array $L$ with polar coordinates (radius and angle) is defined, for each constellation symbol. The algorithm evaluates each element in $L$ individually. The difference is obtained for two threshold variables, $\rho_{T H}$ (radius) and $\alpha_{T H}$ (angle in radians). If the difference is lower in both cases, a cluster is detected. Then, all remaining symbols fulfilling these criteria are removed from $L$, leaving the evaluated symbol as the representative one. It was observed through simulations that the selected symbol hardly affects the final performance. This process is repeated until reaching the end of the array $L$. Simulations have shown that 0.05 and 0.02 are suitable values for $\rho_{T H}$ and $\alpha_{T H}$ respectively. $L$ is then converted back to Cartesian coordinates and the remaining constellation symbols are evaluated for the calculation of the Voronoi regions. In the second step, the Voronoi Regions are calculated, and each region is numbered.
TABLE I

NUMBER OF DISTANCES/NODES. MIMO 2X2 AND 2D-NUC.

\begin{tabular}{c|ccccc}
\hline Demapper & 16NUC & 64NUC & 256NUC & 1kNUC & 4kNUC \\
\hline ML/max-log & 256 & 4096 & 65536 & $1.04 \cdot 10^{6}$ & $1.67 \cdot 10^{7}$ \\
VRS-SFSD & 32 & 100 & 320 & 1124 & 4240
\end{tabular}

The LUT is created and filled by assigning a particular binary value with $b$ bits to each Voronoi region. Finally, in a third step, a morphological filter is applied to the LUT in order to fill the possible gaps between regions.

In a following real transmission, the LUT previously calculated is loaded. For every received symbol, the SFSD demapper needs to perform the quantization step. Then, the received symbol is transformed to the first quadrant, and the corresponding Voronoi region is retrieved from the LUT, as Fig. 3 depicts. Finally, the associated closest symbol is obtained and the SFSD demapping process continues, using the whole constellation. For further information, please refer to [9].

\section{Complexity Reduction And System PERFORMANCE}

\section{A. Complexity Analysis}

As a generic analysis, in this section we consider 2D-NUCs with cardinality points $M$ of 16, 64, 256, 1024 and 4096 for a MIMO system, where 8, 12, 16, 20 and 24 bits per symbol are transmitted, respectively. The complexity is defined as the number of distances or visited nodes to be computed in three different demappers: the optimum ML, max-log and the proposed VRS with SFSD. We assume that the number of transmit and receive antennas, $N_{T}$ and $N_{R}$, is two.

The optimum ML demapper needs to calculate all possible distances. Since it is assumed that only two receive antennas $N_{R}$ are used, the complexity can be obtained as $M^{2}$. The same applies to max-log, since all distances need to be computed. When using VRS and SFSD, the complexity is drastically reduced. The complexity in this case is $M+\log _{2}(M)^{2}$. In a first step, the SFSD algorithm calculates the distances for a single antenna, i.e. $M$ nodes. Then, the ZF-SIC solution is applied to those nodes, reaching the $\log _{2}(M)$ sub-optimal hard solutions. The second term is related to the output LLRs calculated from those solutions [9].

Compared to ML or max-log, the reduction is extremely high. Furthermore, looking up the closest symbol in an already calculated LUT becomes trivial. The number of visited nodes using all considered demappers are shown in Table I. The complexity reduction of VRS with SFSD compared to ML or max-log becomes especially effective for high orders of constellation. The complexity using this method is reduced to $87.5 \%, 97.5 \%, 99.5 \%, 99.8 \%$ and $99.9 \%$, for $16 \mathrm{NUC}, 64 \mathrm{NUC}$, 256NUC, 1024NUC and 4096NUC, respectively.

\section{B. Performance Simulation Results}

As an example of application, we provide some illustrative performance simulation results about the use of VRS with SFSD, compared to ML and max-log demappers, using the 

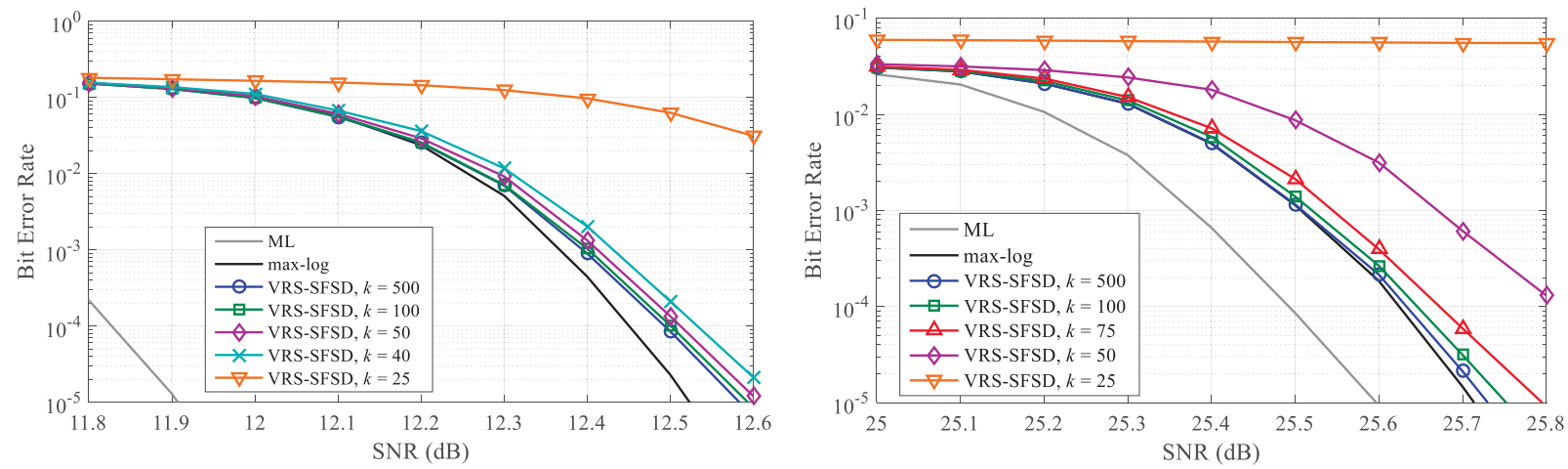

Fig. 4. VRS with SFSD performance as a function of $k$, compared to ML and max-log. 64NUCs are used in each antenna. CRs 5/15 (left) and 13/15 (right).

TABLE II

MINIMUM $k$ AND REQUIRED MEMORY FOR VRS

\begin{tabular}{c|cccc}
\hline & 16NUC & 16NUC & 64NUC & 64NUC \\
& $5 / 15$ & $13 / 15$ & $5 / 15$ & $13 / 15$ \\
\hline$k$ & 18 & 25 & 40 & 75 \\
Number of regions & 4 & 4 & 12 & 16 \\
Memory (Kbits) & 0.65 & 1.25 & 6.40 & 22.50 \\
\hline
\end{tabular}

ATSC 3.0 specification. A low-density parity-check (LDPC) code length of 64800 bits was used. Bit, time and frequency interleavers were employed [1], [6]. We use 16NUCs and 64NUCs with CRs $5 / 15$ and $13 / 15$ in a spatial multiplexing MIMO 2x2 system, under an i.i.d. Rayleigh channel, where co-polar antennas are considered. The entries of the channel matrix are independent amongst each other, with zero-mean. A cross-polar discrimination (XPD) of $6 \mathrm{~dB}$ is assumed, with independent time realizations between symbols [14]. Ideal channel estimation was used. For the VRS method, different values for parameter $k$ were evaluated. Note that the method VRS with SFSD and an infinite $k$ provides max-log performance. Fig. 4 shows the performance of the proposed method for 64NUC with finite $k$ values. As Fig. 4 depicts, a minimum value for $k$ of 40 and 75 is necessary to obtain a performance loss under $0.1 \mathrm{~dB}$ compared to max-log. Two different factors affect the dependency on the CR. With low CRs, the higher error correction capability of the LDPC code allows to cope with a lower number of cells. In addition, the condensation of the NUC reduces the amount of regions to be considered in the VRS quantization of closest symbols. Note that, compared to ML, the proposed method introduces a performance loss of $0.7 \mathrm{~dB}$ for CR $5 / 15$ and $0.2 \mathrm{~dB}$ for CR13/15. Additional results for $16 \mathrm{NUC}$ are depicted in Table II. The number of regions and memory requirements to store the LUT are provided as well. As Table II shows, both the number of LUT cells and memory required increase with constellation order and CR.

\section{CONClusion}

In this paper, an efficient solution for 2D-NUCs and MIMO systems based on Voronoi regions has been proposed. In multiantenna systems, the optimum ML demapping complexity grows exponentially with the number of antennas and the constellation order. To reduce it, suboptimum demappers can be used, such as SFSD. However, SFSD demappers do not work with 2D-NUCs, since they perform a ZF-SIC quantization step that needs to be performed in separated I/Q components. The proposed method quantifies the closest symbol using Voronoi regions and allows SFSD demappers to work. Using VRS and SFSD and compared to ML and max-log demappers, the number of distances to be computed is reduced up to $87.5 \%$, and $97.5 \%$ for $16 \mathrm{NUC}$ and $64 \mathrm{NUC}$ respectively. The highest performance loss introduced is $0.1 \mathrm{~dB}$ compared to max-log and $0.7 \mathrm{~dB}$ compared to ML demappers.

\section{REFERENCES}

[1] L. Michael, and D. Gomez-Barquero, "Bit-Interleaved Coding and Modulation (BICM) for ATSC 3.0," IEEE Trans. Broadcast., vol. 62, no. 1, pp. 181-188, March 2016.

[2] J. Zöllner, and N. Loghin, "Optimization of high-order non-uniform QAM constellations," IEEE International Symposium on Broadband Multimedia Systems and Broadcasting (BMSB), London, UK, June 2013.

[3] D. Gomez-Barquero, C. Douillard, P. Moss, and V. Mignone, "DVBNGH: The next generation of digital broadcast services to handheld devices," IEEE Trans. on Broadcast., vol. 60, no. 2, pp. 246-257, June 2014.

[4] L. Fay, et al., "An overview of the ATSC 3.0 physical layer specification," IEEE Trans. Broadcast., vol.62, no. 1, pp. 159-171, March 2016.

[5] N. Loghin, et al., "Non-Uniform Constellations for ATSC 3.0," IEEE Trans. Broadcast., vol. 62, no. 1, pp. 197-203, March 2016.

[6] D. Gomez-Barquero, et al., "MIMO for ATSC 3.0," IEEE Trans. Broadcast., vol 62, no. 1, pp. 298-305, March 2016.

[7] T. Shitomi, et al., "Field experiments of dual-polarized MIMO-OFDM transmission using non-uniform constellations," IEEE International Conference on Consumer Electronics (ICCE), Istambul, Turkey, Jan. 2016.

[8] T. Shitomi, et al., "Evaluation of simplified demapping algorithm for NUQAMs using urban MIMO channel response," IEEE International Symposium on Broadband and Multimedia Systems and Broadcasting (BMSB), Nara, Japan, June 2016.

[9] L. G. Barbero, T. Ratnarajah, and C. Cowan, "A low-complexity softMIMO detector based on the fixed-complexity sphere decoder," IEEE International Conference on Acoustics, Speech and Signal Processing, Las Vegas, Nevada (USA), March 2008.

[10] T. Kailath, H. Vikalo, and $\mathrm{B}$. Hassibi, MIMO receive algorithms. Cambridge University Press, 2005.

[11] G. H. Golub, and C. F. Van Loan, Matrix computations. The Johns Hopkins Univesity Press, 3rd Edition, 1996.

[12] F. Aurenhammer, "Voronoi Diagrams - A survey of a fundamental geometric data structure," ACM Computing Surveys, vol. 23, no. 3, pp. 345-405, Sept. 1991.

[13] M. Fuentes, D. Vargas, and D. Gomez-Barquero, "Low-complexity demapping algorithm for two-dimensional non-uniform constellations," IEEE Trans. Broadcast., vol. 62, no. 2, pp. 375-383, June 2016.

[14] E. Telatar, "Capacity of multi-antenna Gaussian channels," European Transactions on Telecommunications, vol. 10, no. 6, pp. 585-595, Dec. 1999. 